\title{
Perirhinal input to neocortical layer 1 controls learning
}

One Sentence Summary: Perirhinal cortex gates memory formation in neocortical layer 1

Guy Doron ${ }^{*} \dagger$, Jiyun N. Shin ${ }^{1} \dagger$, Naoya Takahashi ${ }^{1}$, Christina Bocklisch ${ }^{1}$, Salina

Skenderi ${ }^{1}$, Moritz Drüke ${ }^{1}$, Lisa de Mont $^{1}$, Maria Toumazo ${ }^{1}$, Moritz von Heimendahl2 ${ }^{2}$ Michael Brecht ${ }^{2}$, Richard Naud ${ }^{3}$, and Matthew E. Larkum*

†These authors contributed equally to this work

1Institute for Biology, Humboldt University of Berlin, D-10117 Berlin, Germany

2Bernstein Center for Computational Neuroscience, Humboldt University of Berlin, D-10115 Berlin, Germany

${ }^{3}$ uOttawa Brain and Mind Institute, Department of Cellular and Molecular Medicine, University of Ottawa, Ottawa, Ontario, K1H 8M5, Canada

*Corresponding authors: Matthew E. Larkum (matthew.larkum@hu-berlin.de), Guy Doron (guydoron@gmail.com) 


\section{Abstract}

Signals sent back to the neocortex from the hippocampus control the long-term storage of memories in the neocortex ${ }^{1,2}$, but the cellular mechanisms underlying this process remain elusive. Here, we show that learning is controlled by specific medial-temporal input to neocortical layer 1. To show this we used direct cortical microstimulation detection task that allowed the precise region of learning to be examined and manipulated. Chemogenetically suppressing the last stage of the medial temporal loop, i.e. perirhinal cortex input to neocortical layer 1, profoundly disrupted early memory formation but had no effect on behavior in trained animals. The learning involved the emergence of a small population of layer 5 pyramidal neurons $(\sim 10 \%)$ with significantly increased firing involving high-frequency bursts of action potentials that were also blocked by suppression of perirhinal input. Moreover, we found that dendritic excitability was correspondingly enhanced in a similarly-sized population of pyramidal neurons and suppression of dendritic activity via optogenetic activation of dendrite-targeting inhibitory neurons also suppressed learning. Finally, single-cell stimulation of cortical layer 5 pyramidal neurons showed that burst but not regular firing retrieved previously learned behavior. We conclude that the medial temporal input to the neocortex controls learning through a process in $\mathrm{L} 1$ that elevates dendritic calcium and promotes burst firing. 


\section{$1 \quad$ Results}

2

3

4

5

The distributed nature of long-term memory formation in the cortex has challenged research into the underlying mechanisms. For hippocampalindependent learning paradigms there is converging evidence to suggest that cortical layer 1 (L1) is a locus for plasticity ${ }^{3-6}$ involving activity in the distal tuft dendrites of pyramidal neurons that innervate L14,7-9. Far less is known about the mechanisms underlying hippocampal-dependent memory formation in the cortex. Application of the retrograde tracer, Fast Blue, to L1 of primary somatosensory cortex (S1), revealed labeled cells in the deep layers of the perirhinal cortex (Fig. 1a, bottom left). Conversely, expression of ChR2-EYFP via a viral vector (AAV) injected into the deep layers of the perirhinal cortex densely labeled axons in L1 of S1 (Fig. 1a, bottom right), confirming that the perirhinal cortex is the last station in the medial temporal loop before the primary somatosensory neocortex in rodents (Fig. 1a, right and Extended Fig. 1) 2,10,11.

In order to examine the influence of perirhinal cortex on memory formation in neocortex, we adapted a fast-learning, associative and cortexdependent task ${ }^{12}$. Rodents were trained to report short (200 ms) trains of direct electrical microstimulation ( $\mu$ Stim) pulses in layer 5 (L5) of S1 (Fig. 1b) where $\mu$ Stim detection threshold is lowest ${ }^{12}$. Animals initially received a block (5 repetitions) of $\mu$ Stim paired with the reward (sweetened water) regardless of their licking responses. Following a brief pairing period (1-2 blocks), the reward became available if the animal actively licked within a response window of 100$1200 \mathrm{~ms}$ following $\mu$ Stim onset (Fig. 1b). Animals learned this task extremely quickly during the first training session and became experts after about 3 training sessions (see Methods). Ipsilateral injections of lidocaine in the hippocampus showed that this task is hippocampus-dependent (Extended Fig. 2). Making behavior contingent on $\mu$ Stim of $S 1$ allowed us to precisely define the area of interest and the temporal window in order to examine the underlying neuronal mechanisms of memory formation. Moreover, it allowed us to precisely target the perirhinal projection to L1 of S1. We chose a chemogenetic approach to down-regulate synaptic transmission ${ }^{13}$ at the axon terminals of perirhinal long-range projecting neurons without influencing the hippocampus and 
33 parahippocampal regions. Here, we expressed hM4Di receptors (inhibitory designer receptors exclusively activated by a designer drug, DREADD ${ }^{14}$ ) in the perirhinal cortex of mice (Fig. 1c). The axon terminals in S1 were inhibited by application of clozapine-N-oxide (CNO, $10 \mu \mathrm{M})$, injected in to $\mathrm{L} 1$ above the stimulated region (Fig. 1c), 20 mins before training (see Methods).

Specifically blocking perirhinal cortex input to L1 of S1 severely reduced learning during the first training session (Fig. 1d\&e). We quantified learning as the cumulative difference between the number of successful and failed licking responses to $\mu$ Stim ( $\Sigma$ [hits-misses]). By this criterion, mice in which the influence of perirhinal axons on L1 of neocortex was suppressed could not associate the water reward with the $\mu$ Stim over the first training session but rather licked in approximately $50 \%$ of the trials (average learning score $0.48 \pm 0.06$ normalized to the total number of trials, $n=6$ in ctrl versus $-0.03 \pm 0.08, n=7$ in $h M 4 D i / C N O$ treated mice; Wilcoxon rank-sum test, $\mathrm{p}=0.0047)$. Note, CNO alone ${ }^{15}$, i.e. without expression of hM4Di, had no effect on learning ( $n=3$, Wilcoxon rank-sum test, p=0.4; Extended Fig. 2). In contrast to control animals, untrained animals rarely responded to $\mu$ Stim $(-0.54 \pm 0.05, \mathrm{n}=5$ in untrained mice, Wilcoxon rank-sum test, $\mathrm{p}=0.0043$; Fig. 1d\&e). After 3 sessions, trained animals had improved learning scores $(0.87 \pm 0.04$ at session 3 , Wilcoxon rank-sum test, $\mathrm{p}=0.02)$ and this was not affected by suppression of the perirhinal influence on S1 $(0.84 \pm 0.06$, $\mathrm{n}=3$ in CNO-treated trained mice, Wilcoxon sign-rank test, $\mathrm{p}=1$; Fig. 1e and Extended Fig. 2). This suggests that perirhinal cortex is involved in early memory formation but does not affect perception of the $\mu$ Stim per se. The second order somatosensory thalamic area, POm, also projects to L1 in S1 and has been implicated in different learning paradigms ${ }^{3-6,16}$. To examine the influence of POm input in $\mu$ Stim task, this time we expressed hM4Di receptors in POm in Gpr26cre transgenic mice ${ }^{17}$. Suppression of this projection from POm slightly affected learning, however the effect was not significant $(0.25 \pm 0.05, \mathrm{n}=7$, Wilcoxon ranksum test, p=0.18; Extended Fig. 2). Taken together, these results show that the influence of the perirhinal cortex on L1 of the neocortex is crucial for learning the $\mu$ Stim detection task. 
64

65

The results of the chemogenetic experiments (Fig. 1) imply that activity in perirhinal cortex influences activity in S1. Since the $\mu$ Stim electrode was most effective when placed in $L 5^{12}$, we reasoned that the stimulation at least affected L5 neurons. Furthermore, L5 neurons have been implicated in perceptual detection tasks and it has been recently shown that the output of these neurons depends partly on the activation of their apical dendrites that project into L1 18 where the perirhinal inputs arrive. We confirmed ex vivo that perirhinal inputs arriving in L1 synapse on to the tuft dendrites of L5 pyramids (Extended Fig. 3). To investigate the influence of PRh input in S1 activity we made juxtacellular recordings from $\mathrm{L} 5$ in $\mathrm{S} 1$ in the same region as the $\mu$ Stim (Fig. 2a, left). We recorded activity from S1 during learning with and without chemogenetic suppression of perirhinal input to the cortical L1. As in the purely behavioral experiments (see Fig. 1), hM4Di/CNO-treated animals also did not learn the task over the first session $(0.1 \pm 0.04, \mathrm{n}=4$, Wilcoxon rank-sum test against control, $\mathrm{p}=0.04)$. Both the baseline ( $1 \mathrm{~s}$ before $\mu$ Stim) and post-stimulus (0.5-2.5 s after $\mu$ Stim) AP firing rates in L5 pyramidal neurons of these animals $(n=4,52$ cells, 826 trials) was significantly reduced in comparison to control animals (n=2, 28 cells, 706 trials) treated with CNO only (Wilcoxon rank-sum test, $\mathrm{p}<0.001$ for both baseline and post-stimulus; Fig. $2 \mathrm{~b}-\mathrm{d}$ ). These results refer to 'Hit' trials where the animals responded correctly to $\mu$ Stim although we found analogous results in 'Miss' trials (Extended Fig. 3). There was no significant difference in firing rate in control animals between baseline and post-stimulus activity and a slight but significant reduction in hM4Di/CNO-treated animals (see also Extended Fig. 3).

Previously, we found that animals are biased to respond to irregular firing patterns in animals trained on a $\mu$ Stim task ${ }^{19}$ and that burst firing correlates with perceptual detection ${ }^{18}$. We therefore examined burst firing of the same cells during learning in control and hM4Di/CNO-treated animals where learning was blocked. Here, we found that blocking of learning via suppression of perirhinal input significantly decreased both baseline and post-stimulus burst rate (Wilcoxon rank-sum test, $\mathrm{p}<0.001$ ). Interestingly, the burst rate following $\mu$ Stim compared to baseline was greatly increased in control animals (Wilcoxon sign- 
rank test, $\mathrm{p}=0.001$ ) but not in $\mathrm{hM} 4 \mathrm{Di} / \mathrm{CNO}$-treated animals and only in 'Hit' trials (see Extended Fig. 3). We conclude that perirhinal input to L1 mediates learningrelated increases in excitability and burst firing in neocortical L5 neurons.

What information does perirhinal cortex convey during $\mu$ Stim learning? To investigate this, we examined the firing of deep layer neurons in perirhinal cortex (Fig. 2a, right). We found that perirhinal neurons responded robustly to hit trials but not to miss trials after $\mu$ Stim in S1 (Fig. 2f\&g; $n=6$ animals, $n=287$ trials in 28 neurons; firing rate: miss $-8 \pm 11.2 \%$ versus hit $30.9 \pm 11.2 \%, p<0.001$, Wilcoxon rank-sum test). Moreover, the responses in perirhinal cortex included an increase in burst rate compared to baseline only during hit trials (Wilcoxon sign-rank test. $\mathrm{p}<0.001)$. However, the relative change of burst rate between miss and hit trials was not significant (Fig. 2f\&g; burst rate miss $14 \pm 21 \%$ versus hit $55.9 \pm 22.3 \%, \mathrm{p}=0.1$, Wilcoxon rank-sum test). This shows that the perirhinal cortex signals information related to 'Hit' trials primarily via increased AP firing during learning.

It has recently been shown that memory formation is accompanied by an increase in slow cortical oscillations ${ }^{20-23}$. We therefore also analyzed the local field potential (LFP) signals, taken from the same recordings in S1 and perirhinal cortex to assess cortical oscillations during learning. Theta power (4 - $8 \mathrm{~Hz}$ ) in S1 was significantly higher in trained versus untrained animals (Extended Fig. 4; Wilcoxon rank-sum test, $\mathrm{p}<0.0001$ ). Analogously, in perirhinal cortex, we found a significant increase in the theta power in 'Hit' compared to 'Miss' trials during learning (Extended Fig. 4; Wilcoxon rank-sum test, p=0.002). These results suggest that elevated theta power in perirhinal cortex correlates to a transition to elevated theta in response to $\mu$ Stim in S1 in expert animals.

Depolarization of the apical dendrites in L5 pyramidal neurons is shown to reliably lead burst firing behaviour ${ }^{24-26}$. Since learning correlated with an increase in burst firing in these neurons (Fig. 2e) that was dependent on perirhinal input to L1, we hypothesized that the mechanism of learning-induced bursting might involve an enhancement of synaptic influence to the tuft dendrites. We therefore examined $\mathrm{Ca}^{2+}$-dependent activity in the apical 
127

128

129

130

131

132

133

134

135

136

137

138

139

140

141

142

143

144

145

146

147

148

149

150

151

152

153

154

155

156

157

158

dendrites of L5 neurons in S1 using 2-photon microscopy in trained animals. To do this, we expressed GCaMP6f in Rbp4-Cre transgenic mice ${ }^{27}$ and imaged at a depth of $\sim 200 \mu \mathrm{m}$, the region of the apical dendrite known for initiation of dendritic $\mathrm{Ca}^{2+}$ activity ${ }^{7}$ (Fig. 3a-c). Calcium transients measured from $1 \mathrm{~s}$ before the $\mu$ Stim until $3 \mathrm{~s}$ after the $\mu$ Stim in 318 dendrites (Fig. $3 \mathrm{~d} ; \mathrm{n}=4$ mice), revealed three populations with distinct fluorescence profiles (Fig. 3e). A small population (10\%, "ON" dendrites) of dendrites showed substantial increases in fluorescence following $\mu$ Stim with another population (37\%, "OFF" dendrites) of dendrites showing reduced $\mathrm{Ca}^{2+}$ fluorescence. The rest were not responsive to $\mu$ Stim (53\%, "NR" dendrites).

We found similarly distinct and stereotypical output firing patterns in L5 neurons using juxtacellular recordings from trained animals (Fig. 3f; see Extended Fig. 5 for examples). In 11\% of cells we saw a sudden and marked increase in firing $(21.44 \pm 42.16 \mathrm{~Hz})$ briefly following $\mu$ Stim (Fig. 3f; L5 "ON" cells). In another population consisting of $40 \%$ of neurons, there was a decrease in firing $(-6.39 \pm 4.93 \mathrm{~Hz})$ immediately following the $\mu$ Stim (Fig. 3f; L5 "OFF" cells). In most of the cells (49\%), we observed no response to $\mu$ Stim (L5 "NR" cells). Interestingly, the baseline firing rate in L5 ON and L5 OFF cells was significantly higher than in NR cells (Wilcoxon rank-sum test, NR vs. ON: p < 0.0001, NR vs. OFF: $\mathrm{p}<0.0001)$. In contrast to expert animals, we observed low firing rates over all neurons in untrained animals (Extended Fig. 6). Most L5 neurons in untrained animals did not respond to $\mu$ Stim at all $(95 \%, n=63 / 66$ cells) with a small population (5\%, $n=3 / 66$ cells) responding with a small increase $(6.9 \pm 6.30 \mathrm{~Hz})$ briefly after $\mu$ Stim. Taken together with the 2-photon dendritic recordings, we conclude that learning enhances the responsiveness of a small population of L5 pyramidal neurons to apical dendritic input.

To test whether dendritic activity influences learning, we optogenetically activated dendrite-targeting inhibitory neurons during the $\mu$ Stim training. Previous studies have implicated somatostatin (SST) positive interneurons in suppressing plasticity and learning via dendritic inhibition 5,6,8,28,29. We reasoned that if the same circuitry is activated during the $\mu$ Stim detection task, activating SST neurons with channelrhodopsin2 (ChR2) should also impair learning. We 
159 activated SST neurons during training in SST::ChR2 mice using a $500 \mathrm{~ms}$ light 160 pulse starting 300 ms before $\mu$ Stim (Fig. 3g). This abolished learning in a manner 161 almost identical to removing the influence of perirhinal input to L1 (SST: $-0.16 \pm$ 1620.05 , Wilcoxon rank-sum test, $\mathrm{p}=0.002$; Fig. 3h\&i). Notably, continued activation 163 of SST neurons through subsequent training sessions prevented learning over 164 several sessions, unlike block of perirhinal input in which the animals eventually 165 became experts (Extended Fig. 7). Altogether these results suggest that the 166 emergence of a population of neurons underlying learned behavior in the $\mu$ Stim task depends on a dendritic mechanism.

The correlation between both bursting and dendritic activity with 169 learning suggests that bursting might underlie memory retrieval in cortical

170

171

172

173

174

175

176

177

178

179

180

181

182

183

184

185

186

187

188

189

190 neurons. In order to test this hypothesis we devised another learning paradigm in which we first trained animals to respond expertly to $\mu$ Stim and then manipulated the firing of single neurons in S1 using single-cell stimulation ("nanostimulation"12,19,30) via a juxtacellular electrode (Fig. 4a). Expert animals were significantly more likely to lick for reward if bursts of APs (80-120 Hz) were elicited in a single L5 pyramidal neuron of S1 compared to false-positive trials where no current was injected. However, response rate to a train of regularly spiking APs $(30-50 \mathrm{~Hz})$ was not significantly different from falsepositive rate (Fig. 4b\&c; Hit rate; false-positive: 25.94 $\pm 3.6 \%$, regular: $28.11 \pm 4.07 \%$, burst: $31.47 \pm 4.24 \%, n=27$ cells, one-sided paired t-test, $\mathrm{p}=0.03$ ). This indicated that burst firing increased the downstream readout of the firing of a single L5 pyramidal neuron leading to successful behavior. Since the learned behavior could be recovered by burst firing in single pyramidal neurons, these data suggest that burst firing observed in $\mathrm{L} 5^{\mathrm{ON}}$ neurons might be extremely effective in memory recall.

Overall, we have shown that the perirhinal connection to L1 of neocortex is crucial for learning a $\mu$ Stim detection task and involves the conversion of neurons in neocortex to high-firing, burst mode correlated with an increase in dendritic activity (Fig. 4d). This implies that the apical dendrites of L5 neurons are the locus of plasticity related to memory consolidation. This idea is corroborated by our previous study where we showed that stimulus detection in 
191 S1 was dependent on dendritic activity in trained animals ${ }^{18}$ and was disrupted

192 by inhibiting this activity. In addition, dendritic activity is shown to be generated

193 by feedback signals from other cortical areas 7,9 and is enhanced during

194 learning 31 , suggesting that perirhinal input to L1 might serve as a gating signal

195 for the enhancement of cortico-cortical feedback inputs (Fig. 4d). We conclude

196 that the medial temporal input to the neocortex controls learning through a

197 process in L1 that is encoded by dendritic calcium promoting burst firing as the 198 neural signature of memory recall.

199

200

201

202

203

204

205

206

207

208

209

210

211

212

213

214

215

216

217 


\section{References}

219 1. Eichenbaum, H. A cortical-hippocampal system for declarative memory.

220 Nat. Rev. Neurosci. 1, 41-50 (2000).

2212 Rolls, E. T. A computational theory of episodic memory formation in the

222 hippocampus. Behavioural Brain Research 215, 180-196 (2010).

223 3. Letzkus, J. J. et al. A disinhibitory microcircuit for associative fear learning

224 in the auditory cortex. Nature 480, 331-335 (2011).

225 4. Gambino, F. et al. Sensory-evoked LTP driven by dendritic plateau

226 potentials in vivo. Nature 515, 116-119 (2014).

227 5. Abs, E. et al. Learning-Related Plasticity in Dendrite-Targeting Layer 1

228 Interneurons. Neuron 100, 684-699 (2018).

229 6. Williams, L. E. \& Holtmaat, A. Higher-Order Thalamocortical Inputs Gate

230 Synaptic Long-Term Potentiation via Disinhibition. Neuron 101, 91-102

$231 \quad$ (2019).

232 7. $\mathrm{Xu}, \mathrm{N}$. L. et al. Nonlinear dendritic integration of sensory and motor input

233 during an active sensing task. Nature 492, 247-251 (2012).

234 8. Cichon, J. \& Gan, W.-B. Branch-specific dendritic $\mathrm{Ca}^{2+}$ spikes cause

235 persistent synaptic plasticity. Nature 520, 180-185 (2015).

236 9. Manita, S. et al. A Top-Down Cortical Circuit for Accurate Sensory

237 Perception. Neuron 86, 1304-1316 (2015).

238 10. Witter, M. P. \& Groenewegen, H. J. Connections of the parahippocampal

239 cortex in the cat. III. Cortical and thalamic efferents. J. Comp. Neurol. 252,

$240 \quad 1-31(1986)$.

241 11. Agster, K. L. \& Burwell, R. D. Cortical efferents of the perirhinal, postrhinal, 242 and entorhinal cortices of the rat. Hippocampus 19, 1159-1186 (2009). 
243 12. Houweling, A. R. \& Brecht, M. Behavioural report of single neuron

244 stimulation in somatosensory cortex. Nature 451, 65-68 (2008).

245 13. Stachniak, T. J., Ghosh, A. \& Sternson, S. M. Chemogenetic Synaptic

246 Silencing of Neural Circuits Localizes a Hypothalamus $\rightarrow$ Midbrain Pathway

247 for Feeding Behavior. Neuron 82, 797-808 (2014).

248 14. Armbruster, B. N., Li, X., Pausch, M. H., Herlitze, S. \& Roth, B. L. Evolving the

249 lock to fit the key to create a family of G protein-coupled receptors

250 potently activated by an inert ligand. Proc. Natl. Acad. Sci. U. S. A. 104,

$251 \quad 5163-8(2007)$

252 15. Gomez, J. L. et al. Chemogenetics revealed: DREADD occupancy and

253 activation via converted clozapine. Science 357, (2017).

254 16. Audette, N. J., Bernhard, S. M., Ray, A., Stewart, L. T. \& Barth, A. L. Rapid

255 Plasticity of Higher-Order Thalamocortical Inputs during Sensory

256 Learning. Neuron 103, 277-291 (2019).

257 17. Oram, T. B., AhissarE. \& Yizhar, O. Head-motion modulation of the activity

258 of optogenetically tagged neurons in the vibrissal thalamus. Soc Neuro

$259 \quad$ Abstr $736.15(2015)$.

260 18. Takahashi, N., Oertner, T. G., Hegemann, P. \& Larkum, M. E. Active cortical

261 dendrites modulate perception. Science 354, 1587-1590 (2016).

262 19. Doron, G., von Heimendahl, M., Schlattmann, P., Houweling, A. R. \& Brecht,

263 M. Spiking Irregularity and Frequency Modulate the Behavioral Report of

264 Single-Neuron Stimulation. Neuron 81, 653-663 (2014).

265 20. Clouter, A., Shapiro, K. L. \& Hanslmayr, S. Theta Phase Synchronization Is

266 the Glue that Binds Human Associative Memory. Curr. Biol. 27, 3143-3148

267 (2017). 
268 21. Parish, G., Hanslmayr, S. \& Bowman, H. The Sync/deSync Model: How a

269 Synchronized Hippocampus and a Desynchronized Neocortex Code

270 Memories. J. Neurosci. 38, 3428-3440 (2018).

$27122 . \quad$ Schreiner, T., Doeller, C. F., Jensen, O., Rasch, B. \& Staudigl, T. Theta Phase-

272 Coordinated Memory Reactivation Reoccurs in a Slow-Oscillatory Rhythm

273 during NREM Sleep. Cell Rep. 25, 296-301 (2018).

274 23. Hanslmayr, S., Axmacher, N. \& Inman, C. S. Modulating Human Memory via

275 Entrainment of Brain Oscillations. Trends Neurosci. 42, 485-499 (2019).

276 24. Williams, S. R. \& Stuart, G. J. Mechanisms and consequences of action potential burst firing in rat neocortical pyramidal neurons. J. Physiol. 521,

278 467-482 (1999).

279

25. Larkum, M. E., Zhu, J. J. \& Sakmann, B. A new cellular mechanism for coupling inputs arriving at different cortical layers. Nature 398, 338-341 (1999).

26. Larkum, M. E. \& Zhu, J. J. Signaling of layer 1 and whisker-evoked $\mathrm{Ca}^{2+}$ and $\mathrm{Na}^{+}$action potentials in distal and terminal dendrites of rat neocortical pyramidal neurons in vitro and in vivo. J. Neurosci. 22, 6991-7005 (2002).

27. Gerfen, C. R., Paletzki, R. \& Heintz, N. GENSAT BAC Cre-Recombinase Driver Lines to Study the Functional Organization of Cerebral Cortical and Basal Ganglia Circuits. Neuron 80, 1368-1383 (2013).

28. Makino, H. \& Komiyama, T. Learning enhances the relative impact of topdown processing in the visual cortex. Nat. Neurosci. 18, 1116-1122 (2015).

29. Chen, S. X., Kim, A. N., Peters, A. J. \& Komiyama, T. Subtype-specific plasticity of inhibitory circuits in motor cortex during motor learning. Nat. 
293

294

295

296

297

298

299

300

301

302

303

304

305

306

307

308

309

310

311

312

313

314

315

316

317

318

319

Neurosci. 18, 1109-1115 (2015).

30. Houweling, A. R., Doron, G., Voigt, B. C., Herfst, L. J. \& Brecht, M.

Nanostimulation: manipulation of single neuron activity by juxtacellular

current injection. J. Neurophysiol. 103, 1696-1704 (2010).

31. Miyamoto, D. et al. Top-down cortical input during NREM sleep

consolidates perceptual memory. Science 352, 1315-1318 (2016). 
320

321

322

323

325

326

327

329

330

331

332

333

334

335

336

337

338

339

340

341

342

343

344

\section{Methods}

Animals. All experiments and procedures were approved and conducted in accordance with the guidelines given by Landesamt für Gesundheit und Soziales Berlin. The following animal lines were used in this study: C57BL/6J wild-type mice, Gpr26-cre transgenic mice ${ }^{17}$, SST::ChR2 transgenic mice (SST-IRES-Cre mice (JAX stock \#018973) were crossed with Ai32 mice (JAX stock \#024109) ) $)^{32}$ Rbp4-cre transgenic mice ${ }^{27}$ and Wistar rats (Charles River). Male animals were used except for $2 \mathrm{Rbp} 4$-cre mice. The animals were housed in reversed $12 \mathrm{~h}$ light/dark cycle (light on between 21:00 and 09:00) and all the behavioral experiments were performed during dark period of the cycle.

Retrograde and anterograde tracing. For the retrograde labeling of S1 projecting perirhinal neurons, Fast Blue (25\% in $\mathrm{dH}_{2} \mathrm{O}$, Polysciences) soaked in a sterile piece of tissue was applied onto the surface of S1 for $10 \mathrm{~min}$. Incubation time was 7 days before transcardial perfusion. For anterograde tracing and optogenetic ex-vivo experiments AAV-hSyn-hChR2(H134R)-EYFP (Penn Vector Core) was injected in the PRh of $>2$ weeks old C57/BL6 mice. Anesthesia was induced and maintained with isoflurane at 5\% and 2\%, respectively. Mice were placed in a stereotaxic frame and craniotomies were performed using stereotaxic coordinates: anterior-posterior axis (AP) $-1.8 \mathrm{~mm}$, medial-lateral axis (ML) \pm 4.1 $\mathrm{mm}, \mathrm{DV}-4.2 \mathrm{~mm}$ from bregma. Injections were carried out using graduated pipettes broken back to a tip diameter of $10-15 \mu \mathrm{m}$, at a rate of $\sim 0.025 \mu \mathrm{l} / \mathrm{min}$ for a total volume of $0.05-0.07 \mu$ l. Incubation time was at least 3 weeks before transcradial perfusion or ex-vivo experiment. 
345 YFP fluorescence analysis. AAV-hSyn-hChR2(H134R)-EYFP containing acute

346 brain sections were imaged using an Olympus BX51 Microscope with a 4x

347 objective. Fluorescence intensity was quantified with ImageJ software by

348 plotting a line profile across the cortical layers that calculates the brightness

349 value. The average gray value of all images was then normalized to the negative

350 SEM of the lowest grey value across the average line profile.

352 Ex-vivo electrophysiology. After 3-4 weeks of virus expression, sagittal or

353 coronal slices (300 $\mu$ m thick) were prepared from 35-50 day old C57/BL6 mice.

354 Whole-cell patch-clamp recordings were performed from visually identified

355 layer-5 pyramidal neurons using infrared (IR) Dodt-gradient contrast video

356 microscopy. The extracellular solution contained $125 \mathrm{mM} \mathrm{NaCl}, 25 \mathrm{mM} \mathrm{NaHCO}$,

35725 mM Glucose, 3 mM KCl, 1.25 mM NaH2PO4, 2 mM CaCl2, 1 mM MgCl2, pH 7.4

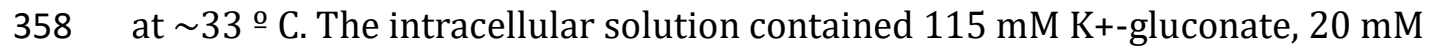

$359 \mathrm{KCl}, 2 \mathrm{mM}$ Mg-ATP, 2 mM Na2-ATP, 10 mM Na2-phosphocreatine, 0.3 mM GTP,

$36010 \mathrm{mM}$ HEPES, $0.05 \mathrm{mM}$ Alexa 594 and biocytin (0.2\%), pH 7.2. Whole-cell

361 voltage recordings were performed from the soma (4-6 $\mathrm{M} \Omega$ ) using a Multiclamp

$362700 \mathrm{~b}$ (Molecular devices) amplifier. Data was acquired with an ITC-18 board and

363 analyzed using Igor software. Optogenetic synaptic stimulation was performed

364 via an LED (470 nm) (2 ms pulses) located in L1 around the tuft dendrite. To

365 activate $\mathrm{hM}_{4} \mathrm{D}_{\mathrm{i}}$ receptor, Clozapine-N-Oxide (CNO) (Tocris Bioscience) was bath

366 applied (final concentration $10 \mu \mathrm{M}$ ).

367

368 Chemogenetic manipulation of PRh axonal activity. Mice ( $>4$ weeks) were

369 anesthetized with ketamine/xylazine (13 $\left.\mathrm{mg} \mathrm{kg}^{-1} / 1 \mathrm{mg} \mathrm{kg}^{-1}\right)$ by intraperitoneal 
370 injection (i.p.). Animals were kept on a thermal blanket during entire surgery

371 and recovery. Lidocaine (1\%, wt/vol, AlleMan Pharma) was injected around the

372 surgical site before the scalp incision. The periosteum was removed and small

373 craniotomy was made on the injection sites. Injection coordinates for PRh were

374 AP $-1.8 \mathrm{~mm}, \mathrm{ML} \pm 4.2 \mathrm{~mm}, \mathrm{DV}-4.2 \mathrm{~mm}$ and for POm were AP $-2 \mathrm{~mm}, \mathrm{ML}+1.2$

375 mm, DV -3.0 mm from bregma. AAV1/2-hSyn1-hM4D(Gi)-mCherry-WPRE-

376 hGHp(A) (Viral Vector Facility of the University of Zurich) was injected either

377 bilaterally ( $\mathrm{n}=16$ for PRh and $\mathrm{n}=7$ for Pom) or unilaterally ( $\mathrm{n}=3$ for PRh) $(0.15$ -

$378 \quad 0.20 \mu \mathrm{l}$ per side). Further experiments were performed after 3 weeks of

379 expression.

380 In order to activate hM4Di receptor, CNO dissolved in extracellular

381 solution $(10 \mu \mathrm{M})$ was applied into superficial layers of S1 (initial depth at 150

$382 \mu \mathrm{m}$ and over the day injection pipette was advanced (up to $300 \mu \mathrm{m}$ ) in order to

383 compensate tissue growth on the craniotomy) at least $20 \mathrm{~min}$ before the

384 microstimulation training. CNO was applied into two adjacent sites (150 $\mu \mathrm{l}$ each)

385 of the craniotomy to maximize the CNO diffusion area.

387 Headpost implant and head-restraint habituation. A lightweight aluminum

388 head-post (mouse) or a metal bolt (rat) was implanted on the skull of the animal

389 under ketamine/xylazine anaesthesia (13 $\mathrm{mg} \mathrm{kg}^{-1} / 1 \mathrm{mg} \mathrm{kg}^{-1}$ for mice, $100 \mathrm{mg} \mathrm{kg}^{-}$

$3901 / 5 \mathrm{mg} \mathrm{kg}^{-1}$ for rats, i.p.). For mice used in chemogenetic experiments, the

391 implantation was performed > 10 days after viral injection. After the scalp and

392 periosteum were removed, a thin layer of light curing adhesives (OptiBond, Kerr

393 and Charisma, Kulzer) was applied to the skull. A head-post was fixed on the

394 skull on the left hemisphere with a dental cement (Paladur, Heraeus Kulzer). 

implantation. Habituation time at the first day was 5 min and then gradually

397 increased each day until the animal sat calmly for $1 \mathrm{~h}$. Animals were water

398 restricted from the second day ( $1 \mathrm{ml} /$ day) of the habituation and then trained to

399 receive the saccharin (Sigma-Aldrich) water (0.5\% for mice and $0.1 \%$ for rats)

400 from the licking port. Licking was monitored using a piezo-based sensor attached

401 to the licking port. Weight and health of the animal were monitored daily.

402 Habituation for head restraint and licking typically took 5 days.

403 Two to three days before the microstimulation training or/and

404 juxtacellular recording, $1.5 \mathrm{~mm} \times 1.5 \mathrm{~mm}$ craniotomy was made on the right

405 barrel cortex centered at AP $1.25 \mathrm{~mm}$ and ML $3.75 \mathrm{~mm}$ from bregma for mice

406 and AP $2.5 \mathrm{~mm}$ and ML $5.5 \mathrm{~mm}$ from bregma for rats. For perirhinal cortex

407 recording in rats, craniotomy was made on AP $4.5 \mathrm{~mm}$ and ML $5.0 \mathrm{~mm}$ from

408 bregma. Then a recording chamber was implanted for chronic access to this

409 region. The dura was left intact and the craniotomy was covered with silicon

410 (Kwik-Cast, World Precision Instruments).

412 Optogenetic manipulations. For optogenetic activation of SST neurons,

413 SST::ChR2 transgenic mice that express ChR2 in SST-positive cells were used.

414 Photostimulation light (465 nm, $2 \mathrm{~mW}, 500$-ms pulse starting at $300 \mathrm{~ms}$ before

415 stimulus onset) was delivered via the optic fiber placed above the craniotomy.

416 To prevent the mice from distinguishing photostimulation trials from control

417 trials using visual cues, the recording chamber was covered with a black rubber

418 to prevent light leakage from photostimulation into the animals' eyes. 
420 Microstimulation detection task. Animals were trained to perform

421 microstimulation task as described elsewhere ${ }^{12,19,33}$. Briefly, animals were

422 trained to respond with tongue lick to a $200 \mathrm{~ms}$ train of microstimulation pulses

423 applied to barrel cortex (40 cathodal pulses at $200 \mathrm{~Hz}, 0.3 \mathrm{~ms}$ pulse duration)

424 through a tungsten microelectrode (Microprobes) in depth of $\sim 700 \mu \mathrm{m}$ (mice) or

$425 \sim 1500 \mu \mathrm{m}$ (rats) from pia and presented at random intervals. In the first session,

426 initial intensity of $160 \mu \mathrm{A}$ pulses were injected into the cortex and paired with a

427 drop of water reward (pairing period). After 5 pairings, testing began where

428 animals were rewarded only if they lick the licking port within 100 to $1,200 \mathrm{~ms}$

429 after stimulus onset. Tongue lick responses were detected with piezo-based

430 sensor (mice) or beam breaker (rats). The time of the first lick after stimulus

431 onset was taken as the reaction time. To encourage animals to use a

432 nonconservative response criterion, we only mildly punished licks in the

433 interstimulus interval with an additional $1.5 \mathrm{~s}$ delay to the next stimulus

434 presentation. Once animals reached $80 \%$ hit rate, pulse intensity was gradually

435 decreased during and over the sessions until it reached $10 \mu \mathrm{A}$ (mice) or $5 \mu \mathrm{A}$

436 (rats). Control mice reached to $10 \mu \mathrm{A}$ within 3-5 days of training. Expert in this

437 study means animals who performed the task with $>80 \%$ hit rate at the $10 \mu \mathrm{A}$

438 (mice) or $5 \mu \mathrm{A}$ (rats).

440 In vivo juxtacellular recording. Following head-restraint habituation,

441 juxtacellular recordings were performed from deep layer neurons from S1 and

442 PRh in awake head-fixed animals during $\mu$ Stim detection task. The glass pipette

443 (4-8 $\mathrm{M} \Omega$ ) for juxtacellular recording during microstimulation task was filled

444 with extracellular solution containing: $135 \mathrm{mM} \mathrm{NaCl}, 5.4 \mathrm{mM} \mathrm{KCl}, 1.0 \mathrm{mM} \mathrm{MgCl} 2$, 
$4451.8 \mathrm{mM} \mathrm{CaCl} 2$ and $5 \mathrm{mM}$ HEPES (pH 7.2). The juxtacellular signal was amplified

446 and low-pass filtered at $3 \mathrm{kHz}$ by a patch-clamp amplifier (NPI) and sampled at

$44725 \mathrm{kHz}$ by a Power1401 data acquisition interface under the control of Spike2

448 software (CED). For PRh recording in rats, the pipette was inserted with $17^{\circ}$

449 toward lateral and $50^{\circ}$ toward anterior. The mean depth in juxtacellular

450 recording of S1 in mice was $1156.8 \pm 25.56 \mathrm{~mm}$ and of PRh in rats was 6339.64

$451 \pm 122.07 \mathrm{~mm}$, which is likely an overestimate of the true depth due to oblique

452 penetrations and dimpling.

453

454 Two-photon $\mathrm{Ca}^{2+}$ imaging. For in vivo two-photon calcium imaging, AAV2/1-

455 Syn-Flex-GCaMP6f-WPRE (Penn Vector Core) was injected through a glass

456 pipette (tip diameter, 5-10 $\mu \mathrm{m}$ ) into the left S1 barrel cortex on the basis of

457 stereotaxic coordinates (AP -1.5 mm and ML $3.2 \mathrm{~mm}$ from bregma). A single

458 injection (100 nl) was made at $700 \mu \mathrm{m}$ deep from the pial surface. Three weeks

459 after the injection, a 3-mm craniotomy was made over the injection site and

460 sealed with a 3-mm glass coverslip (\#1) with cyanoacrylate glue. A light-weight

461 head-post was fixed on the skull in the right hemisphere with light-curing

462 adhesives and a dental cement. Habituation of mice to head restraint and

463 following imaging experiment begin 4 weeks after the virus injection.

464 Imaging from behaving mice was performed with a resonant-scanning

465 two-photon microscope (Thorlabs) equipped with GaAsP photomultiplier tubes

466 (Hamamatsu Photonics). GCaMP6f was excited at $940 \mathrm{~nm}$ (typically 30-40 mW

467 at the sample) with a Ti:Sapphire laser (Mai Tai eHP Deep See, Spectra-Physics)

468 and imaged through a 16×, 0.8 NA water immersion objective (Nikon). Full-

469 frame images (256 × 256 pixels $)$ were acquired from apical dendrites of L5 
470 neurons expressing GCaMP6s at a depth of 150-200 $\mu \mathrm{m}$ at $58.6 \mathrm{~Hz}$ using

471 ScanImage 4.1 software (Vidrio Technologies). Tungsten electrodes for

472 microstimulation was inserted through the access port on the chronic glass

473 window.

474

475 Histology. Animals were perfused transcardially with phosphate-buffered saline

476 (PBS) followed by 4\% paraformaldehyde (PFA). Brains were removed and post-

477 fixed for $>24 \mathrm{~h}$. Coronal sections $(150 \mu \mathrm{m}$ thick $)$ were collected using a

478 vibratome. For DAPI staining, NucBlue (Invitrogen) was applied to sections in

479 PBS for $10 \mathrm{~min}$. The sections were imaged with an epifluorescence microscope or

480 a confocal microscope.

482 Single-Neuron Stimulation Detection Task. Once rats performed at current

483 intensities below $5 \mu \mathrm{A}$ on 2 consecutive days, we switched to single-cell

484 stimulation experiments, as previously described ${ }^{12,33}$. Briefly, the animals were

485 head fixed during the task, and waited for the microstimulation/nanostimulation

486 detection task to begin, which it did when a neuron was found. The glass pipette

487 for juxtacellular single-cell stimulation and recording was glued to a tungsten

488 microelectrode used for microstimulation at a distance of $\sim 70 \mathrm{~mm}$, as described

489 elsewhere ${ }^{12,33}$. The glass pipette was filled with intracellular solution containing:

490135 mM K-gluconate; 10 mM HEPES; 10 mM Na2-phosphocreatine; 4 mM KCl; 4

491 mM MgATP; and 0.3 mM Na3GTP (pH 7.2). Recording depth was 1902 \pm 60.73

$492 \mathrm{~mm}$, which is likely an overestimate of the true depth due to oblique

493 penetrations and dimpling. 
494 During single-cell stimulation trials, a fixed duration square-wave current pulse 495 was injected into a neuron through a glass pipette. Every stimulation sequence

496 contained each step exactly once, while their order was varied pseudo-randomly

497 from trial to trial. To induce a regular spike pattern, we used a single $100 \mathrm{~ms}$ DC

498 current step. To elicit burst like spike pattern, brief stimulation duration of 25

499 ms was used, followed by 1175 ms inhibition at current intensities of $50 \%$ used

500 in the nanostimulation, to prevent any further spikes during the stimulation trial.

501 Single-cell stimulation trials, catch trials without current injection and

502 microstimulation trials were pseudo randomly interleaved in series of 6 trials

503 including 3 microstimulation trials, 2 single-cell stimulation trials (each of

504 different duration) and 1 catch trial. All trials were presented at random

505 intervals (Poisson process, mean 3 s). Microstimulation currents were adjusted

506 (range 3-8 $\mu$ A, mean $4.2 \pm 1.1 \mu$ A (s.d.)) such that animals performed close to the

507 detection threshold, resulting in an average microstimulation hit rate of $90 \%$.

508

509 Data analysis and statistics. Recorded neurons were separated into putative

510 fast-spiking (FS) interneurons and regular-spiking (RS) pyramidal neurons

511 based on spike half-width and firing rate. Cells with spike half-width lower than

$5120.5 \mathrm{~ms}$ and firing rate higher than $8 \mathrm{~Hz}$ were classified to FS. Only RS were used

513 for further analysis.

514 All the cells and trials recorded over days were pooled together for

515 comparing activity (firing rate or burst rate) changes during miss and hit trials.

516 Bursts were identified as at least two spikes with an inter-spike interval of $\leq 15$

517 ms. Time window between 1 and $0 \mathrm{~s}$ before the stimulus ([-1 0$] \mathrm{s})$ was used to

518 calculate the baseline activity and $0.5-2.5 \mathrm{~s}([+0.5+2.5] \mathrm{s})$ after stimulus was 
519 used to calculate post-stimulus activity. For firing rate and burst rate change

520 analysis, difference between pre-stimulus frequency and post-stimulus

521 frequency was divided by average pre-stimulus frequency.

$522 \quad$ All analysis for $\mathrm{Ca}^{2+}$ imaging was performed using imageJ and custom

523 written codes in Matlab. Horizontal and vertical drifts of imaging frames due to

524 animal motion were corrected by registering each frame to a reference image

525 based on whole-frame cross-correlation. The reference image was generated by

526 averaging any given consecutive 100 frames in which motion drifts were

527 minimal. Regions of interest (ROIs) for apical dendrites of L5 neurons were

528 manually selected with the help of average intensity and standard deviation

529 projections across movie frames. For each ROI, pixel values inside the ROI were

530 averaged to obtain the time series of $\mathrm{Ca}^{2+}$ fluorescence. The extracted signals

531 were corrected for neuropil contamination by subtracting the local, peri-

532 dendritic neuropil signals. Fluorescence change $\left(\Delta F / F_{0}\right)$ was calculated as

$533\left(F-F_{0}\right) / F_{0}$, where $F_{0}$ was the baseline fluorescence value in the ROI throughout

534 the whole imaging session.

535 For local field potential (LFP) analysis, juxtacellularly recorded voltages

536 were band-pass filtered at $4-30 \mathrm{~Hz}$ and a power spectrum was calculated using

537 the Stockwell Transform ${ }^{34,35}$, over a $2 \mathrm{~s}$ period before stimulus onset and $5 \mathrm{~s}$

538 period afterwards. In order to avoid potential artifacts caused by stimulation, the

539 analysis was restricted to microstimulation or nanostimulation trials where the

540 following trial occurred more than $5 \mathrm{~s}$ after stimulus onset. The power spectrum

541 was calculated separately for individual trials and its absolute magnitude

542 averaged within the different response categories (hits and misses). To obtain 
543 the population spectra for the different response categories the power spectra of

544 individual trials were then averaged.

545 For the classification of cells, peri-stimulus time-histograms (PSTHs)

546 were calculated for each cell by averaging spikes in time bins of $100 \mathrm{~ms}$ for times

547 within 2 seconds of hit-trials. For each cell, the stationary rate and standard

548 deviation were computed based on the PSTHs in the period $[-2,0] \mathrm{s}$. Cells were

549 classified to ON cell or OFF cell if PSTHs in the period [0.3,0.4] s was either more

550 than $3^{*}$ standard deviation (SD) above the stationary rate, or less than $3 *$ SD

551 below, respectively. Other cells were classified as NR cells. Similarly, dendrites

552 were classified into ON, OFF and NR dendrites.

553 Unless otherwise stated, all values are indicated as mean \pm SEM. Shapiro-

554 Wilk test was performed to test normality of the data. For non-parametric test,

555 significance was determined using Wilcoxon signed-rank test within group and

556 Wilcoxon rank-sum test between groups at a significance level of 0.05 . No

557 statistical tests were run to predetermine sample size, and blinding and

558 randomization were not performed.

559

560

561

562

563

564

565

566

567 


\section{References}

569 32. Madisen, L. et al. A toolbox of Cre-dependent optogenetic transgenic mice

570 for light-induced activation and silencing. Nat. Neurosci. 15, 793-802

$571 \quad$ (2012).

572 33. Voigt, B. C., Brecht, M. \& Houweling, A. R. Behavioral detectability of single-

573 cell stimulation in the ventral posterior medial nucleus of the thalamus. J.

$574 \quad$ Neurosci. 28, 12362-7 (2008).

575 34. Stockwell, R. G., Mansinha, L. \& Lowe, R. P. Localization of the complex

576 spectrum: the S transform. IEEE Trans. Signal Process. 44, 998-1001

577 (1996).

578 35. Ari, S., Das, M. K. \& Chacko, A. ECG signal enhancement using S-Transform.

579 Comput. Biol. Med. 43, 649-660 (2013).

580

581

582

583

584

585

586

587

588

589

590

591

592

593 
a

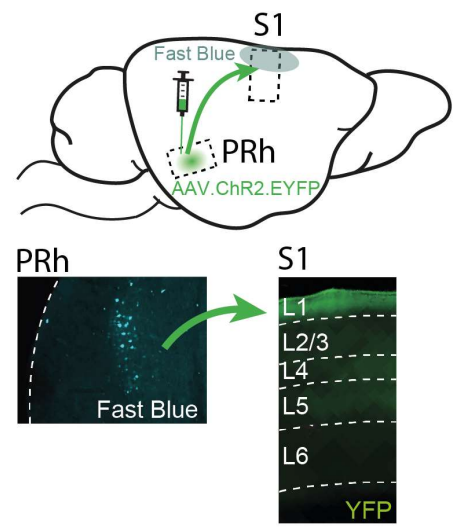

595

596

597

598

599

600

601

602

603

604

605

606

607

608

609

610
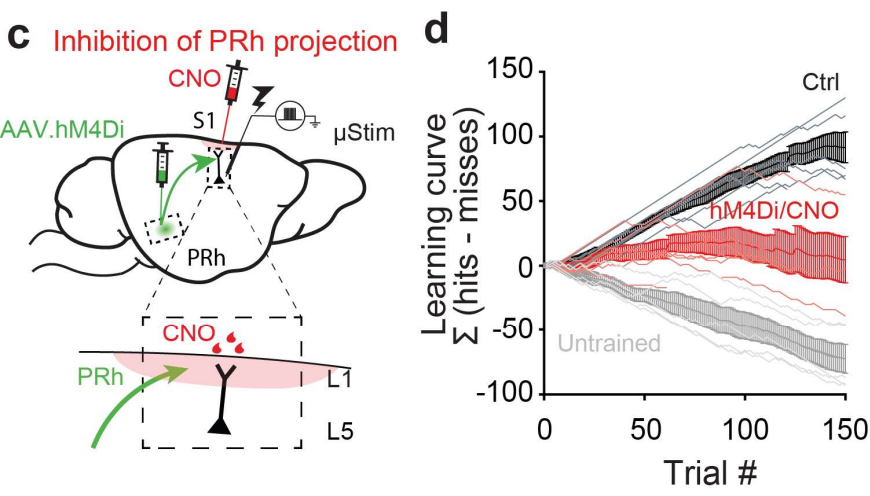

b

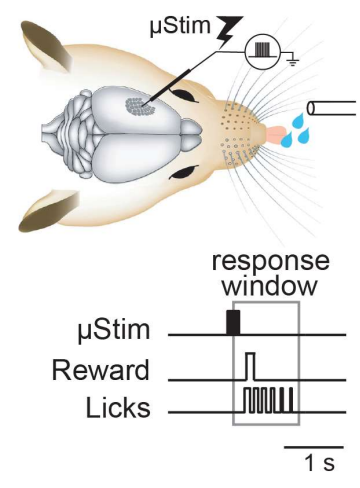

e

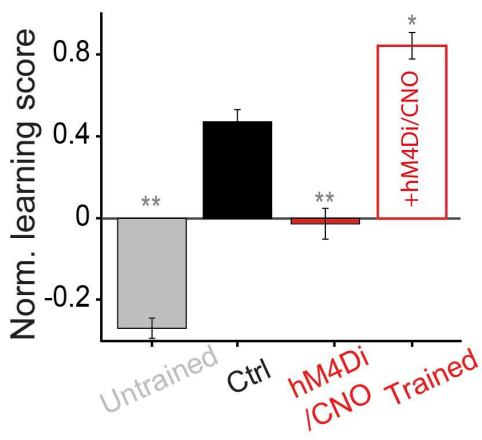

Learning

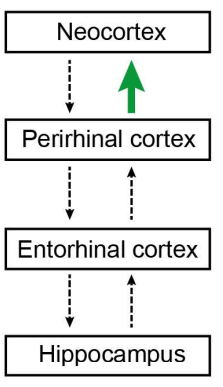

Figure 1 | Perirhinal projection to neocortical L1 is necessary for learning a

$\boldsymbol{\mu S t i m}$ task. a, Left, sagittal view of the rodent brain showing perirhinal projection to primary somatosensory cortex (S1). Lower left, retrograde tracing. Deep layer neurons in perirhinal cortex (PRh) were labeled with Fast Blue after application of dye to L1 of S1. Lower right, anterograde tracing. ChR2/EYFP labeled axons of PRh project strongly to L1 of S1. Right, simplified connectivity map between the neocortex, perirhinal cortex, entorhinal cortex and hippocampus. Investigated connection is highlighted in green. Note that not all the connections are shown here. b, Schematic of $\mu$ Stim detection task. Tungsten electrode was placed in L5 of S1. Animals learned to respond by licking within a 1.1-s window following $\mu$ Stim. c, Schematic of chemogenetic silencing of PRh axons in L1 of S1 during $\mu$ Stim task. AAV.hM4Di was injected to PRh and CNO was applied in superficial layer of S1 before $\mu$ Stim task (see Methods). Inset, enlarged view of superficial layer of S1. Red shade represents CNO effective area. d, Cumulative learning curve of control mice (black), mice with PRh axonal 
611 suppression (hM4Di; red) and untrained mice (grey) during 150 trials in the first

612 session. Light lines represent individual mouse. Bold lines with error bars

613 represent the mean and SEM, respectively, of each group. e, Last value of

614 cumulative learning curve normalized by total number of trials (Norm. learning

615 score) during learning and after learning (Trained). Wilcoxon rank-sum test

616 against control, ${ }^{*} \mathrm{p}<0.05,{ }^{* *} \mathrm{p}<0.01$.

617

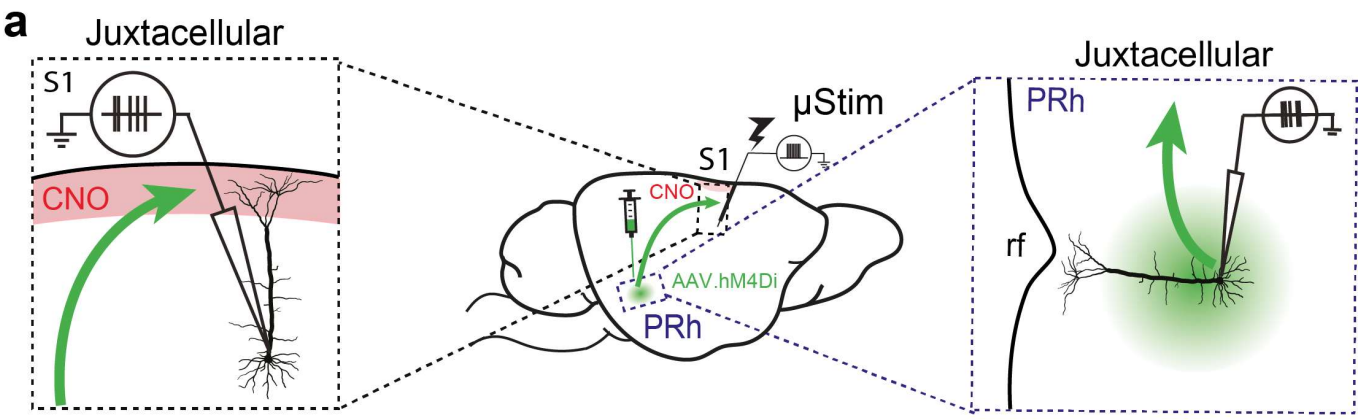

b

b Control S1 Hit

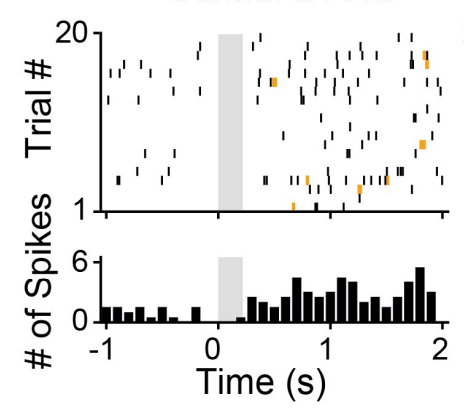

d

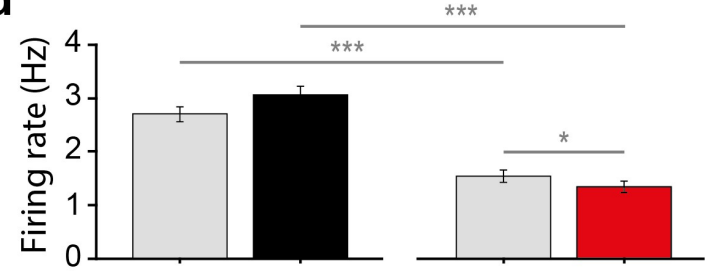

e

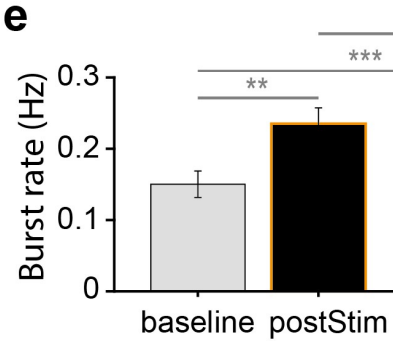

C $\mathrm{hM} 4 \mathrm{Di} / \mathrm{CNO} \mathrm{S} 1 \mathrm{Hit}$
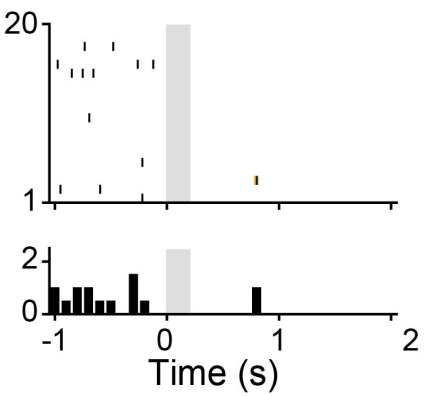

***

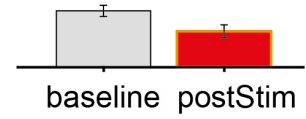

f
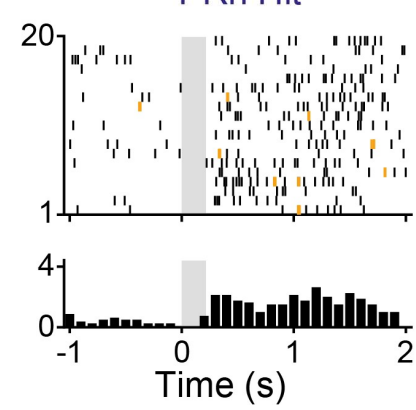

g

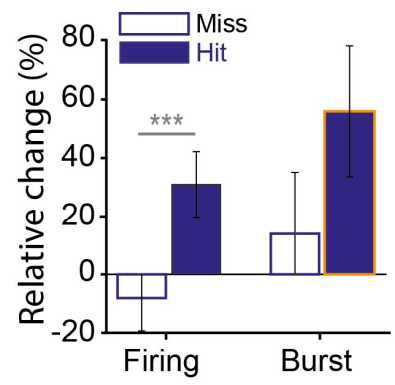


620 Figure 2 | Learning-induced burst firing in layer 5 pyramidal neurons in S1

621 is PRh-dependent. a, Schematic of juxtacellular recording of L5 pyramidal

622 neurons in S1 (left) and deep layer pyramidal neurons in PRh (right). Red shade:

623 CNO targeted area, rf: rhinal fissure. b, c, Representative raster plot (upper) and

624 PSTH (lower) during hit trials in L5 pyramidal neurons in control S1 and hM4Di

625 S1, respectively. Bursts are marked by yellow ticks in the raster plot. Gray box:

$626 \mu$ Stim. Note that y-axis scales differ for visibility. d, e Firing rate and burst rate

627 during hit trials in control S1 and in hM4Di S1, respectively. f, Representative

628 raster plot (upper) and PSTH (lower) during hit trials in perirhinal neuron. g,

629 Relative change of firing rate and burst rate during miss and hit trials from PRh

630 neurons. ${ }^{*} \mathrm{p}<0.05,{ }^{* *} \mathrm{p}<0.01,{ }^{* * *} \mathrm{p}<0.001$.

a

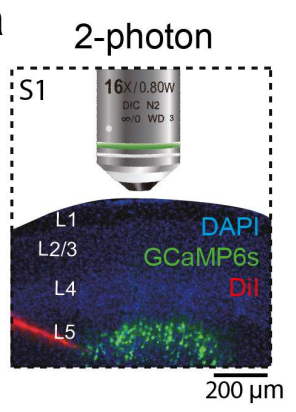

b

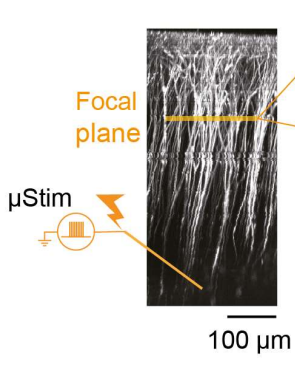

C

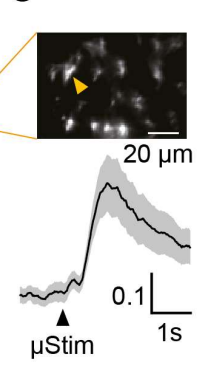

d

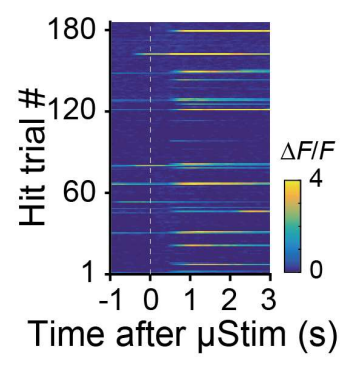

e

Dendrites

$\mathbf{f}$

Soma

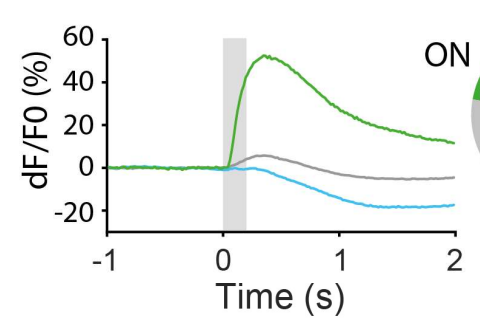

g

Optogenetic activation of SST+ INs

631

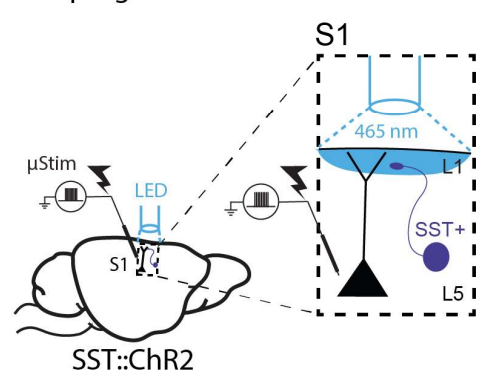

h

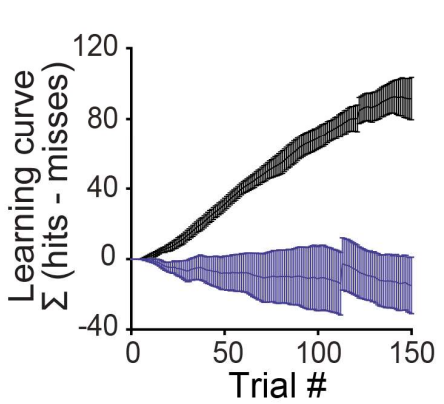

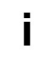

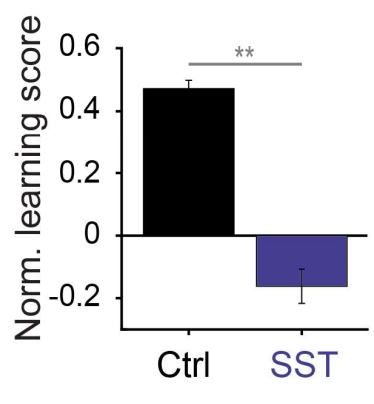


632 Figure 3 | Dendritic activity-dependent emergence of distinct L5

633 subpopulations after learning. a, Two-photon $\mathrm{Ca}^{2+}$ imaging from the apical

634 dendrites of L5 pyramidal neurons in Rbp4-cre mice during $\mu$ Stim task. DiI

635 shows the location of $\mu$ Stim electrode. $\mathbf{b}, \mathrm{Z}$-stack image of recorded dendrites

636 and $\mu$ Stim electrode in L5. c, Horizontal imaging plane (upper) $(\sim 200 \mu \mathrm{m}$ from

637 pia) and average $\mathrm{Ca}^{2+}$ responses (lower) for all trials from a dendrite marked

638 with a yellow arrow. $\mathbf{d}, \mathrm{Ca}^{2+}$ responses in an apical dendrite marked in c. during

639180 trials of $\mu$ Stim task. e, Left, Average peri-stimulus time $\mathrm{Ca}^{2+}$ responses in $\mathrm{ON}$,

640 OFF and NR dendrites (total $n=318$ dendrites) during hit trials (See Methods for

641 classification criteria). Gray box: $\mu$ Stim. Right, the fraction of ON, OFF and NS

642 dendrites. f, Left, Average PSTH of L5 ${ }^{\mathrm{ON}}$, L5 OFF and L5 ${ }^{\mathrm{NR}}$ neurons (total $\mathrm{n}=272$

643 cells) during hit trials (See Methods for classification criteria). Gray box: $\mu$ Stim.

644 Right, the fraction of L5 ${ }^{\mathrm{ON}}$, L5 OFF and L5 ${ }^{\mathrm{NR}}$ neurons. g, Schematic of optogenetic

645 activation of SST+ interneurons during $\mu$ Stim task in SST::ChR2 mice. Blue light

$646(465 \mathrm{~nm})$ was shed on the surface of the craniotomy (See Methods). Inset,

647 magnified view of the targeted circuit in S1. h, Cumulative learning curve of

648 control $(n=6$, black) and SST::ChR2 mice $(n=6$, dark blue) during the first session.

649 i, Normalized learning score of control and SST::ChR2 mice at the first session.

650 Wilcoxon rank-sum test, ${ }^{* *} \mathrm{p}<0.01$.

651 

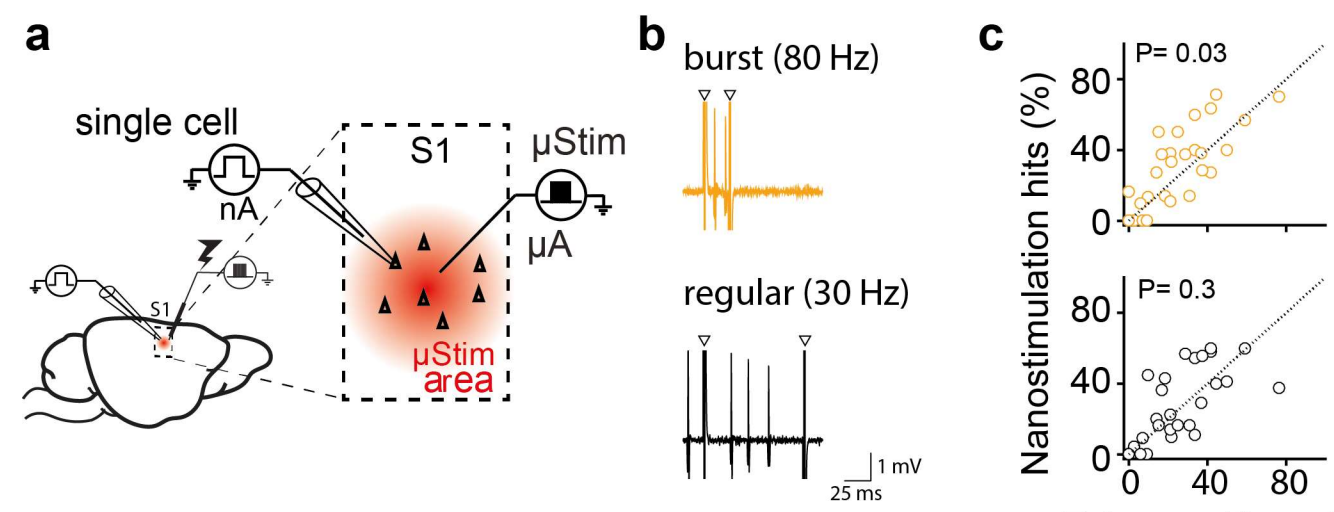

False positives (\%)

d

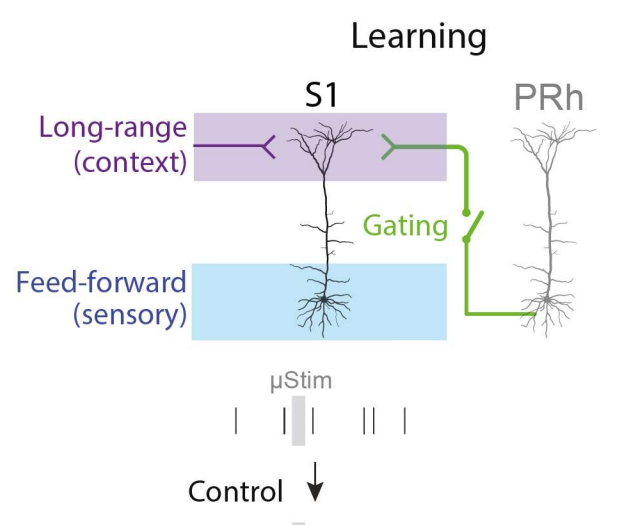

652

653 Figure 4 | Burst firing in single $L 5$ pyramidal neurons can retrieve learned

654 behavior. a, Schematic of single-cell stimulation. Inset, $\mu$ Stim stimulates a 655 population of neurons surrounding electrode (red shade) and glass electrode 656 stimulates a single neuron (triangle). b, Current injection protocol to induce 657 either regular AP firing ( $30 \mathrm{~Hz}$, black) or high frequency $(80-120 \mathrm{~Hz}$, yellow) 658 bursts in single cells. c, Response rates (hits) for regular AP firing (black) or 659 burst firing (yellow) trials versus false-positive trials ( $\mathrm{n}=27$ cells). One-sided 660 paired t-test. d, Gating theory of memory formation in cortex. PRh inputs to L1 661 gate long-range inputs that modulate the firing mode of L5 pyramidal neurons in 662 S1 and learning. 